\title{
A Synthesis of Electronically Controllable Current-Mode PI, PD and PID Controllers Employing CCCDBAs
}

\author{
Somchai Srisakultiew ${ }^{1,2}$, Montree Siripruchyanun ${ }^{2 *}$ \\ ${ }^{1}$ Department of Computer Engineering, Faculty of Engineering and Architecture, Rajamangala University of \\ Technology Isan, Nakhonratsima, Thailand \\ ${ }^{2}$ Department of Teacher Training in Electrical Engineering, Faculty of Technical Education, King Mongkut's University of \\ Technology North Bangkok, Bangkok, Thailand \\ Email: ${ }^{*}$ mts@kmutnb.ac.th
}

Received March 21, 2013; revised April 30, 2013; accepted May 7, 2013

Copyright (C) 2013 Somchai Srisakultiew, Montree Siripruchyanun. This is an open access article distributed under the Creative Commons Attribution License, which permits unrestricted use, distribution, and reproduction in any medium, provided the original work is properly cited.

\begin{abstract}
This paper presents a synthesis of current-mode PI, PD and PID controllers employing current controlled current differential buffer amplifiers (CCCDBAs). The features of these controllers are that: the output parameters can be electronically/independently controlled by adjusting corresponding bias currents in the proportional, integral, and deviation controllers; circuit description of the PID controller is simply formulated, it consists of four CCCDBAs cooperating with two grounded capacitors, and PI and PD controllers are composed of three CCCCDBAs and a grounded capacitor. Without any external resistor, the proposed circuits are very suitable to develop into integrated circuit architecture. The given results from the PSpice simulation agree well with the theoretical anticipation. The approximate power consumption in a closed loop control system consisting of the PI, PD and PID controller with low-pass filter passive plant are $4.03 \mathrm{~mW}, 4.85 \mathrm{~mW}$ and $5.71 \mathrm{~mW}$, respectively, at $\pm 1.5 \mathrm{~V}$ power supply voltages.
\end{abstract}

Keywords: Current-Mode; PID Controller; CCCDBA

\section{Introduction}

The proportional-integral-derivative (PID) controllers are the most important control devices employed in industrial process control [1]. Classical implementations of the PID controller contain several active elements to realize the transfer function. For instance, parallel structure using operational amplifiers (Op-Amp) [2] requires four sections: Proportional (P), Integral (I), Derivative (D) transfers and adder. Proportional-integral (PI), Proportional-derivative (PD) and PID controllers with adjustable parameters are implemented in various pieces of work. These controllers are used in many applications, for example, motor speed controllers, temperature controllers, fluid controllers and etc. $[3,4]$.

For the last two decades, the attention is subsequently focused on the PID controllers using different high-performance active building blocks such as, operational transconductance amplifiers (OTAs) [5,6], current feedback op-amp (CFAs) [7,8], second generation current conveyors (CCIIs) [9-13], second generation current

\footnotetext{
"Corresponding author.
}

controlled current conveyors (CCCIIs) [14-16], and current differencing buffered amplifiers (CDBAs) [17]. The literature surveys show that a large number of circuit realizations for PI, PD and PID controllers simulators have been reported [5-17]. Unfortunately, these reported circuits suffer from one or more of following weaknesses:

- Excessive use of the active and/or passive elements [5-17];

- Circuit requirement external resistors [5,6,7-17];

- Lack of electronic tenability [7-13,17];

- Absence of independent control of their parameters $[5,7,9,14,15]$.

The current differencing buffered amplifier (CDBA) is a reported active component especially suitable for a class of analog signal processing [18]. The fact that the device which can operate in both current and voltage modes provides flexibility and enables a variety of circuit designs. In addition, it can offer advantageous features such as high-slew rate, free from parasitic capacitances, wide bandwidth and simple implementation [19]. However, the CDBA cannot be controlled by the parasitic 
resistances at two current input ports so when it is used in a circuit, it must unavoidably require some external passive components, especially the resistors. This makes it not appropriate for IC implementation due to occupying more chip area, higher power dissipation and cannot electronic controllable. Subsequently, Maheshwari and Khan have proposed the modified-version CDBA whose parasitic resistances at two current input ports can be controlled by an input bias current and it is newly named current controlled current differencing buffered amplifier (CCCDBA) [20].

Presently, a current-mode technique has been more popular than the voltage-mode one. This is due to operating in the low-voltage environment found portable and battery-powered equipment. Since a low-voltage operating circuit has become necessary, the current-mode technique is ideally suited for this purpose more than the voltage-mode one. Furthermore, there is a growing interest in synthesizing the current-mode circuits because of their unique potential advantages such as larger dynamic range, higher signal bandwidth, greater linearity, simpler circuitry, and lower power consumption [21].

The purpose of this paper is to introduce a synthesis of current-mode PI, PD and PID controllers employing CCCDBAs. The features of the proposed controllers are that: the output parameter can be electronically/independently controlled by adjusting corresponding bias currents in the proportional, integral, and deviation controllers: circuit description of the PID controller is very simple, consisting of four CCCDBAs cooperating with two grounded capacitors. PI and PD controller consists of three CCCDBAs cooperating with a grounded capacitor. The simulations are performed by PSpice to exhibit the performance of the developed controllers.

\section{Theory and Principle}

\subsection{Basic Concept of Current Controlled Current Differencing Buffered Amplifier (CCCDBA)}

Since the proposed circuits are based on CCCDBAs, a brief review of CCCDBA is given in this section. Basically, the CCCDBA is composed of translinear elements, mixed loops and complementary current mirrors. Generally, its properties are similar to the conventional CDBA [19], except that input voltages of CCCDBA are not zero and the CCCDBA has finite input resistances $R_{p}$ and $R_{n}$ at the $p$ and $n$ input terminals, respectively. These intrinsic resistances are equal and can be controlled by the bias current $I_{B}$ as shown in the following equation

$$
\left[\begin{array}{c}
V_{p} \\
V_{n} \\
I_{z 1, z 2} \\
V_{w}
\end{array}\right]=\left[\begin{array}{cccc}
R_{p} & 0 & 0 & 0 \\
0 & R_{n} & 0 & 0 \\
1 & -1 & 0 & 0 \\
0 & 0 & 1 & 0
\end{array}\right]\left[\begin{array}{c}
I_{p} \\
I_{n} \\
V_{z} \\
I_{w}
\end{array}\right],
$$

For BJT CCCDBA, the input resistances; $R_{p}$ and $R_{n}$ can be expressed to be

$$
R_{p}=R_{n}=\frac{V_{T}}{2 I_{B}} .
$$

$V_{T}$ and $I_{B}$ are the thermal voltage and input bias current, respectively. The symbol and the equivalent circuit of the CCCDBA are illustrated in Figures 1(a) and (b), respectively.

\subsection{Synthesis of Proposed Controllers Employing CCCDBAs}

\subsubsection{PI Controller}

PI controller is composed of a proportional and an integral term. The PI controller is sufficient when the process dynamics is an essentially first-order system. The proposed PI controller employs three CCCDBAs, a grounded capacitor as shown in Figure 2. Transfer function of general PI controller: $H_{\mathrm{PI}}(s)$ can be written as (3).

$$
H_{\mathrm{PI}}(s)=\frac{I_{O}}{I_{\text {in }}}=K_{P i}+\frac{1}{T_{i} s} \text {. }
$$

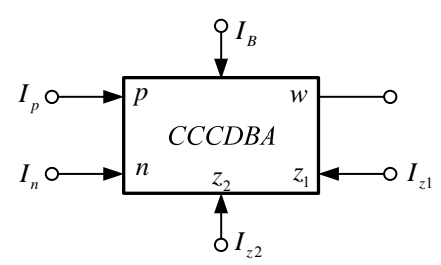

(a)

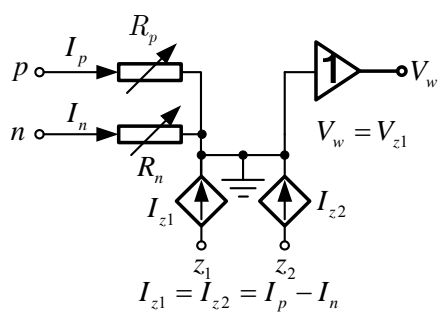

(b)

Figure 1. The CCCDBA (a) symbol (b) equivalent circuit.

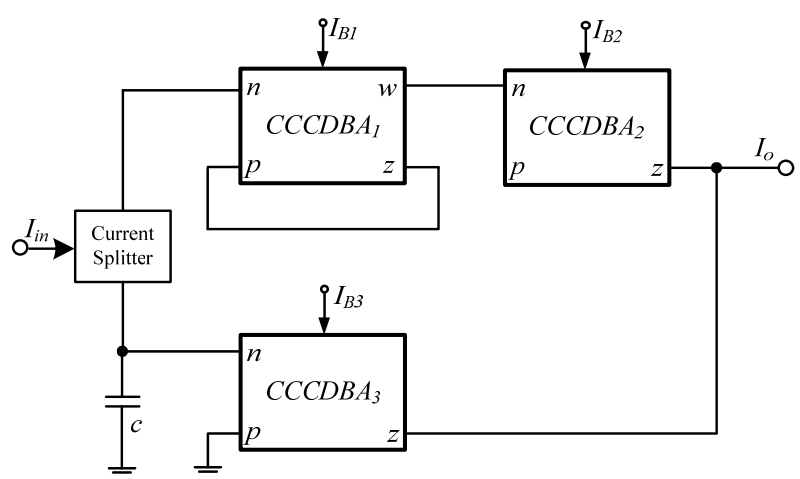

Figure 2. The proposed PI controller. 
The CCCDBA based PI controller in Figure 2 has the transfer function as

$$
H_{\mathrm{PI}}(s)=\frac{I_{O}}{I_{\text {in }}}=\frac{I_{B 2}}{2 I_{B 1}}-\frac{C R_{n}}{s} .
$$

Substituting $R_{n}=\frac{V_{T}}{2 I_{B}}$ into (4), it yields

$$
H_{\mathrm{PI}}(s)=\frac{I_{O}}{I_{\text {in }}}=\frac{I_{B 2}}{2 I_{B 1}}-\frac{2 V_{T} C}{s I_{B 3}} .
$$

From (5), it is found that $H_{\mathrm{PI}}$ and $T_{i}$ can be independently controlled by $I_{B 1} / I_{B 2}$ and $I_{B 3}$, respectively.

\subsubsection{PD Controller}

The PD controller is the most widely used in strategy for robot manipulators, motor speed control, and etc. Additionally, more advanced controllers often incorporate to PD algorithms in their control-loop to reach the desired configuration. The derivative term of the PD controller deals with slope of error, and it is effective in the transient-response. The derivative term has no effect if the steady-state error is constant in a corresponding time. The proposed PD controller employs three CCCDBAs, one grounded capacitor shown in Figure 3. The general transfer function of PD controller: $H_{\mathrm{PD}}(s)$ can be written in (6)

$$
H_{\mathrm{PD}}(s)=\frac{I_{O}}{I_{\text {in }}}=K_{P d}+T_{d} s .
$$

The CCCDBAs based PD controller shown in Figure 3 has the transfer function as (7)

$$
H_{\mathrm{PD}}(s)=\frac{I_{O}}{I_{\text {in }}}=\frac{I_{B 2}}{2 I_{B 1}}+R_{n} s C .
$$

Substituting $R_{n}=\frac{V_{T}}{2 I_{B}}$ into (7), it yields

$$
H_{\mathrm{PD}}(s)=\frac{I_{O}}{I_{\text {in }}}=\frac{I_{B 2}}{2 I_{B 1}}+\frac{V_{T} s C}{2 I_{B 3}} .
$$

From (8), it can be seen that the $K_{P}$ can be electronically controlled by either $I_{B 1}$ or $I_{B 2}$ and $T_{D}$ parameter can be adjusted by adjust $I_{B 3}$ with independent each other.

\subsubsection{PID Controller}

Proportional-integral-derivative (PID) controllers are extensively used in industry. It is estimated that more than $90 \%$ of all control loops involve PID controllers, where the proportional term adjusts the speed response of the system, the integral term adjusts the steady-state error of the system and the derivative term adjusts the degree of stability of the system.

The proposed current-mode PID controller is shown in Figure 4. It consists of only four CCCDBAs and two

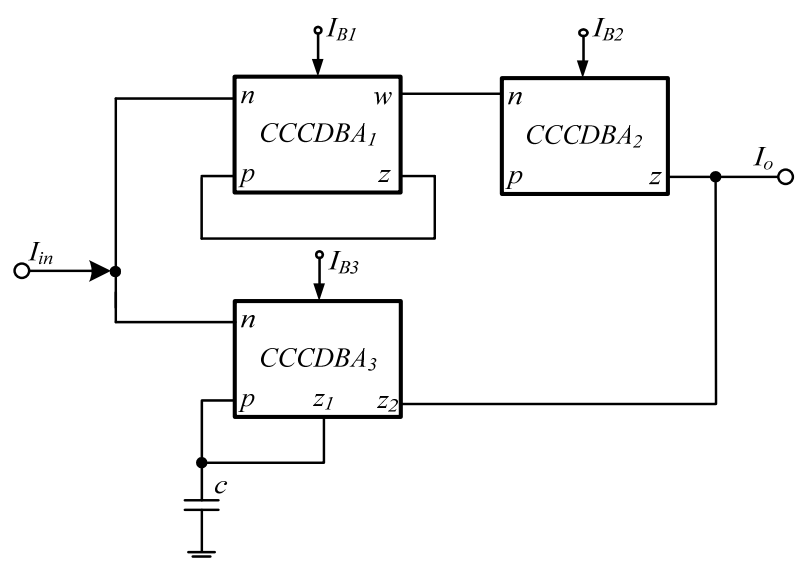

Figure 3. The proposed PD controller.

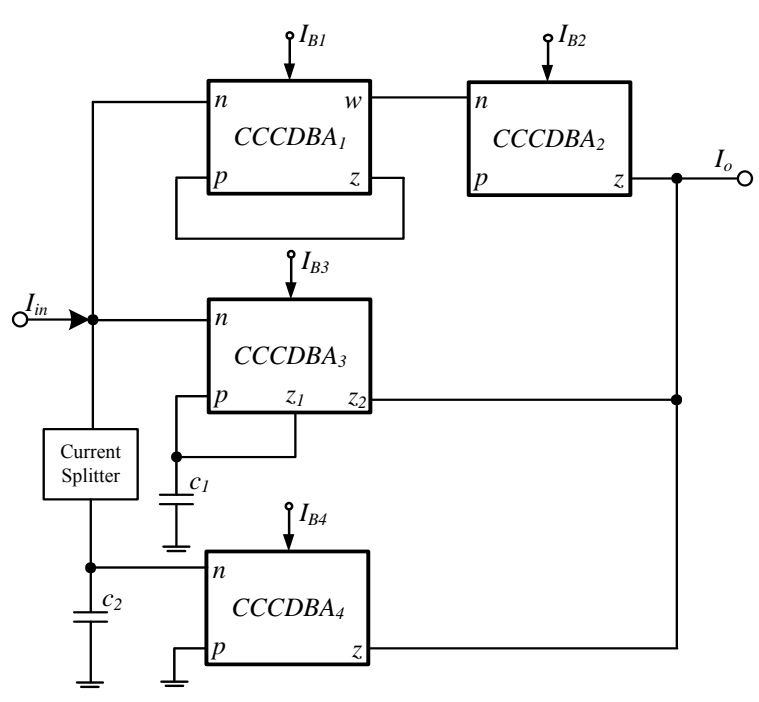

Figure 4. The proposed PID controller.

grounded capacitors. The transfer function of general analog PID controller: $H_{\mathrm{PID}}(s)$ can be written as depicted in (9), where $K_{p}$ is the proportional gain, $K_{i}$ is the integral time, and $K_{d}$ is the derivative time parameters

$$
\begin{aligned}
& H_{\mathrm{PID}}(s)=\frac{I_{\text {out }}(s)}{I_{\text {in }}(s)}=K_{p}+\frac{K_{i}}{s}+s K_{d}, \\
& H_{\mathrm{PID}}(s)=\frac{K_{d} s^{2}+K_{p} s+K_{i}}{s} .
\end{aligned}
$$

The transfer function of the proposed PID controller will be shown by

$$
H_{\mathrm{PID}}(s)=\frac{I_{O}}{I_{i n}}=\frac{I_{B 2}}{2 I_{B 1}}-\frac{C_{2} R_{n 4}}{s}+s C_{1} R_{n 3} .
$$

Substituting $R_{n i}=\frac{V_{T}}{2 I_{B i}}$ into (10), we obtain

$$
H_{\mathrm{PID}}(s)=\frac{I_{O}}{I_{i n}}=\frac{I_{B 2}}{2 I_{B 1}}-\frac{V_{T} C_{2}}{s 2 I_{B 4}}+\frac{V_{T} s C}{2 I_{B 3}} .
$$


From (11), the PID controller's parameters can be assigned to the required values by adjusting the corresponding $I_{B}$. Additionally, it can be seen that the PID parameters $\left(K_{p}, K_{i}\right.$ and $\left.K_{d}\right)$ can be independently controlled by $I_{B 1} / I_{B 2}, I_{B 3}$ and $I_{B 4}$, respectively.

\section{Simulation Results}

To prove the performances of the proposed controllers, the PSpice simulation program was used for the examinations. The PNP and NPN transistors employed in the proposed circuits were simulated by using the parameters of the PR200N and NR200N bipolar transistors of the ALA400 transistor array from AT\&T [22]. Figure 5 depicts schematic description of the CCCDBA used in the simulations. These proposed circuits were biased with corresponding input bias current $I_{B}$ of the CCCDBAs with the symmetrical $\pm 1.5 \mathrm{~V}$ supplies voltages.

To validate the practical application of the proposed controllers, firstly, the proposed current-mode PID controllers and passive low-pass filter were used to realize a closed-loop control system as depicted in Figure 6. For the current-mode low-pass filter, the circuit is shown in Figure 7 with an additional output terminal. The current transfer function of the low-pass filter is found to be

$$
H_{l p}=\frac{I_{o}}{I_{\text {in }}}=\frac{C R_{1}}{L C s^{2}+\left(R_{1}+R_{2}\right) s C+1} .
$$

The passive elements of the filter in Figure 7 were determined by $R_{1}=R_{2}=1 \mathrm{k} \Omega, L=1 \mu \mathrm{H}$ and $C=1 \mu \mathrm{F}$. Figure 8 shows frequency response of passive low-pass filter employed as a plant of closed-loop control system.

Figure 9 illustrates the simulation results of the PI controller using CCCDBAs without the plant, where $C=$ $1 \mathrm{nF}$, input signal of $20 \mu \mathrm{A}$ step waveform. A transient response of the PI controller is shown in Figure 9(a). Figure 9(b) demonstrates that the $K_{P i}$ of the PI controller can be adjusted. The $I_{B 2}$ of PI controller is tuned to 13 $\mu \mathrm{A}, 26 \mu \mathrm{A}$ and $52 \mu \mathrm{A}$ where $I_{B 1}=13 \mu \mathrm{A}, I_{B 3}=26 \mu \mathrm{A}$ and $C=1 \mathrm{nF}$. On the other hand, the integral conditions variation with $I_{B 3}$ to $13 \mu \mathrm{A}, 26 \mu \mathrm{A}$ and $52 \mu \mathrm{A}$ is shown in Figure 9(c), it can be verified that the integral conditions of the PI controller can be electronically/independently tuned by $I_{B 3}$, as depicted in (5).

Figure 10 shows the simulation results of the proposed PD controller without a closed-loop control system, where $C=470 \mathrm{nF}, I_{B 1}=13 \mu \mathrm{A}, I_{B 2}=26 \mu \mathrm{A}$ and $I_{B 3}=26$ $\mu \mathrm{A}$, input signal of $20 \mu \mathrm{A}$ step waveform. The simulation result as shown in Figure 10(a) is a transient response of the PD controller. Figure 10(b) demonstrates that the $K_{\mathrm{PD}}$ of the PD controller can be tuned. The $I_{B 2}$ of PD controller is adjusted to $26 \mu \mathrm{A}, 52 \mu \mathrm{A}$ and $104 \mu \mathrm{A}$, where $I_{B 1}=26 \mu \mathrm{A}, I_{B 3}=26 \mu \mathrm{A}$ and $C=470 \mathrm{nF}$. On the other hand, the derivative condition variation with $I_{B 3}$ to $26 \mu \mathrm{A}, 52 \mu \mathrm{A}$ and $104 \mu \mathrm{A}$ is shown in Figure 10(c). It

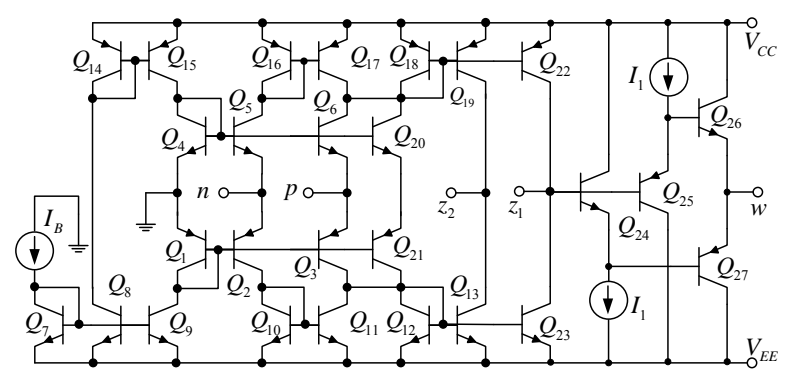

Figure 5. Internal construction of CCCDBA.

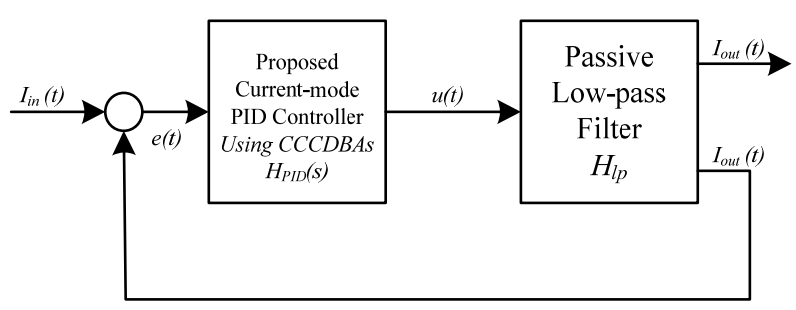

Figure 6. A closed-loop control system.

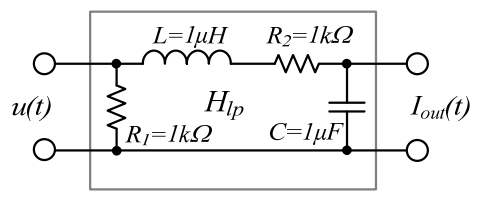

Figure 7. The passive RLC low-pass filter used as a plant.

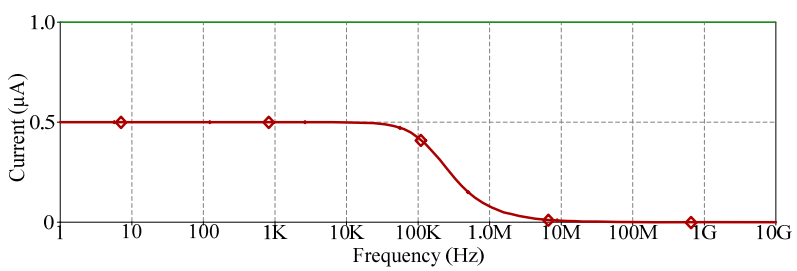

Figure 8. Frequency response of RLC low-pass filter plant.

can be verified that the differential conditions of the PD controller can be electronically/independently tuned by $I_{B 3}$, as depicted in (8).

To obtain the proposed current-mode PID controller, whose transfer function of $K_{p}=1, T_{i}=38.46 \mu \mathrm{s}$ and $T_{d}=$ $235 \mu \mathrm{s}$, we use the passive elements as followed: $C_{1}=1$ $\mu \mathrm{F}, C_{2}=1 \mathrm{nF}$, where $I_{B 1}=20 \mu \mathrm{A}, I_{B 2}=40 \mu \mathrm{A}, I_{B 3}=50$ $\mu \mathrm{A}$ and $I_{B 4}=20 \mu \mathrm{A}$. We determine input signal as a step waveform of $20 \mu \mathrm{A}$ at $1 \mathrm{kHz}$ of frequency. Figure 11(a) shows the result of the transient response for an initial condition obtained from the closed-loop control system in Figure 6. Finally, Figure 11(b) shows the relationship between input and output signals during a steady state condition. The power consumption of the closed loop control systems is $5.71 \mathrm{~mW}$.

\section{Conclusion}

In this study, a synthesis of novel current-mode PI, PD 


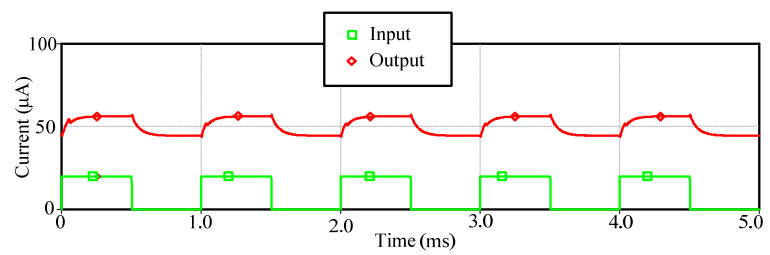

(a)

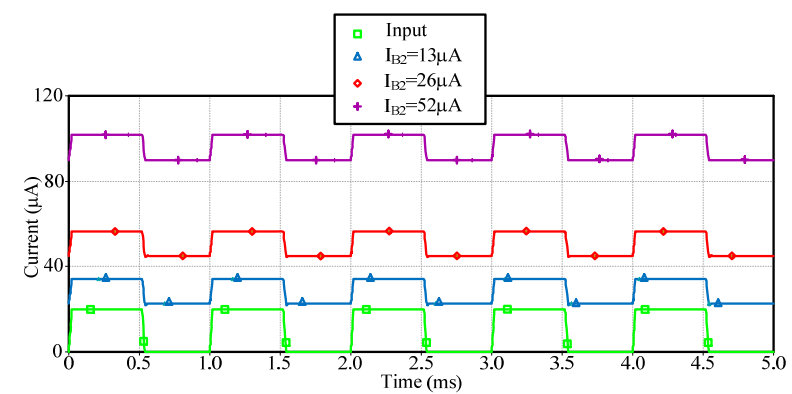

(b)

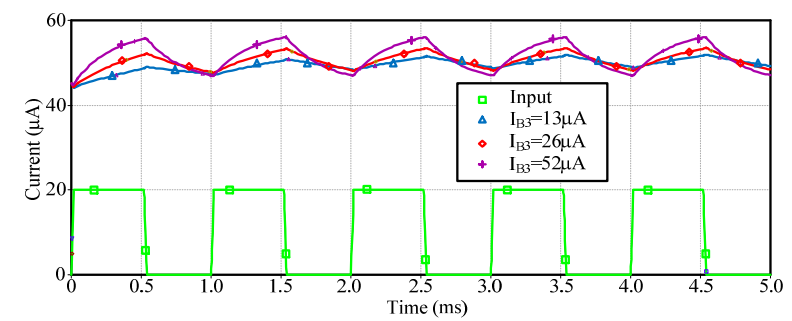

(c)

Figure 9. Simulation results of proposed PI controller.

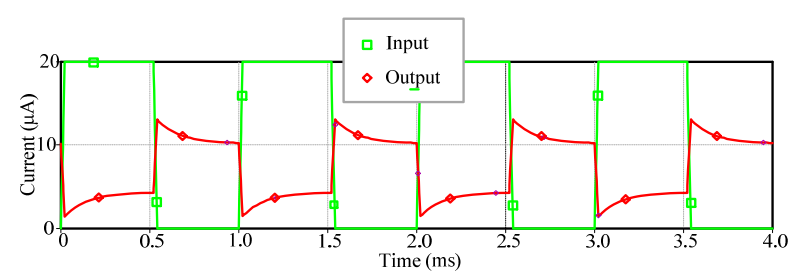

(a)

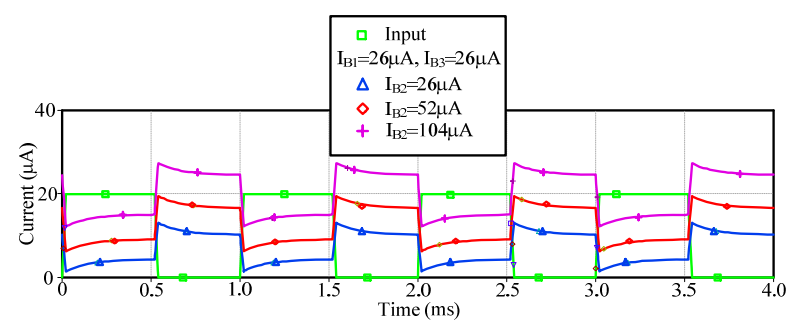

(b)

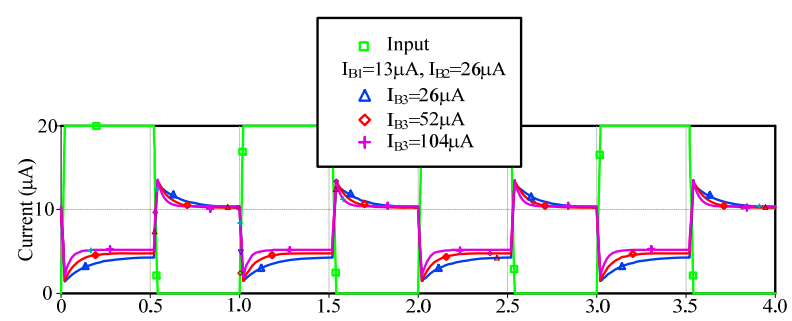

(c)

Figure 10. Simulation results of proposed PD controller.

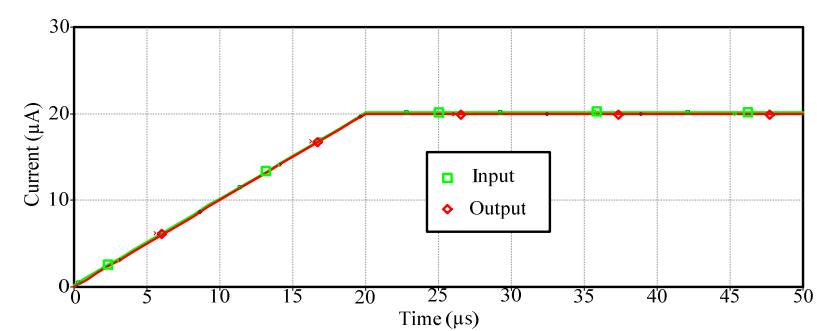

(a)

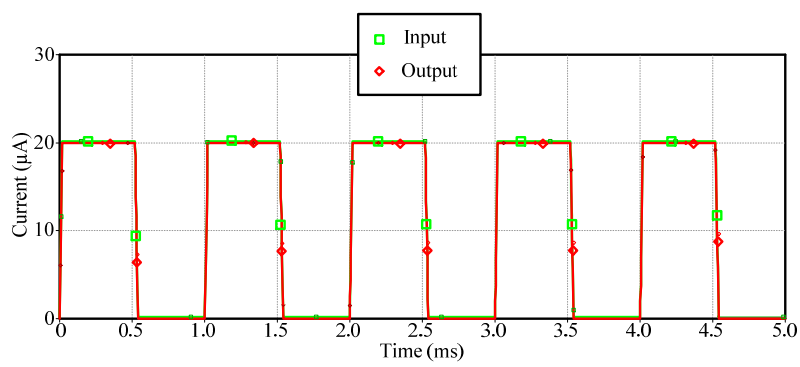

(b)

Figure 11. Input and output relationship of PID controller with closed-loop control system.

and PID controllers were realized by employing CCCDBAs. All PI, PD and PID parameters can be tuned electronically and independently by the corresponding bias currents. The circuit description of the PID controller comprises only four CCCDBAs and two grounded capacitors. PI and PD controller consists of three CCCDBAs cooperating with a grounded capacitor, without any external resistor. Simulation results confirm the theoretical analysis. It is easily modified to use in control systems using a microcontroller [20]. With mentioned features, it is very suitable to realize the proposed controllers in a monolithic chip for use in battery-powered electronic devices.

\section{REFERENCES}

[1] A. J. Michael and H. M. Mohammad, "PID Control New Identification and Design Methods," Springer, Berlin, 2005.

[2] S. Franco, "Design with Operational Amplifiers and Analog Integrated Circuits," McGraw-Hill, New York, 1997.

[3] B. C. Kuo, “Automatic Control System," Prentice-Hall, Upper Saddle River, 1997.

[4] "Designing Ultrafast Loop Response with Type-III Compensation for Current Mode Step-Down Converters," Ap. Note SLVA352, Texas Instrument, 2010. http://focus.ti.com/lit/an/slva352/slva352.pdf

[5] C. Edral, A. Toker and C. Acar, "OTA-C Based Proportional-Integral-Derivative (PID) Controller and Calculating Optimum Parameter Tolerances," Turkish Journal of Electrical Engineering and Computer Sciences, Vol. 9, No. 2, 2001, pp. 189-198.

[6] S. Srisakultiew, S. Lawanwisut and M. Siripruchyanun, "A Synthesis of Electronically Controllable Current-Mode PI, PD and PID Controllers Employing OTAs," The Pro- 
ceeding of $10^{\text {th }}$ International Prince of Songkla University (PSU) Engineering Conference, Songkhla, 14-15 May 2012.

[7] E. Cevat, "A New Current-Feedback-Amplifiers (CFAs) Based Proportional-Integral-Derivative (PID) Controller Realization and Calculating Optimum Parameter Tolerances," Pakistan Journal of Applied Sciences, Vol. 2, No. 1, 2002, pp. 56-59. doi:10.3923/jas.2002.56.59

[8] S. Srisakultiew, S. Lawanwisut and M. Siripruchyanun, "A Synthesis of Current-Mode PI, PD and PID Controllers Employing CFAs," The Proceeding of $51^{\text {th }}$ Kasetsart University Annual Conference, Bangkok, February 2013, pp. 144-151.

[9] S. Minaei, E. Yuce, S. Tokat and O. Cicekoglu, "Simple Realization of Current-Mode and Voltage-Mode PID, PI and PD Controllers," Proceedings of the IEEE International Symposium on Industrial Electronics, Dubrovnik, 20-23 June 2005, pp. 195-198.

[10] E. Cevat, A. Toker and C. Acar, "A New ProportionalIntegral-Derivative (PID) Controller Realization by Using Current Conveyor and Calculating Optimum Parameter Tolerances," Journal of Electrical and Electronics, Vol. 1, 2001, pp. 267-273.

[11] J. A. Svoboda, "Current Conveyors, Operational Amplifiers and Nullors," IEE Proceedings, Vol. 136, No. 6, 1989, pp. 317-322.

[12] S. Minaei, E. Yuce, S. Takat and O. Cicekoglu, "Simple Realizations of Current-Mode and Voltage-Mode PID, PI and PD Controller," Proceeding of the IEEE International Symposium on Industrial Electronics, Vol. 1, 2005, pp. 195-198.

[13] V. Srikul, S. Srisakultiew and M. Siripruchyanun, "A Synthesis of Current-Mode PI, PD and PID Controllers Employing CCIIs," Proceeding of Electrical Engineering Network 2012 of Rajamangala University of Technology, Nong Khai, April 2012, pp. 513-516.

[14] C. Erdal, H. Kuntman and S. Kafali, "A Current Con- trolled Conveyor Based Proportional-Integral-Derivative (PID) Controller," Journal of Electrical \& Electronics Engineering, Vol. 4, No. 2, 2004, pp. 1248-1248.

[15] W. Naksup, V. Kiranon, J. Vongdektum and V. Sangpisit, "PID, PI and PD Controllers Based on CCCII," Proceeding of $31^{\text {st }}$ Electrical Engineering Conference, Nakhonnayok, 2009, pp. 927-930.

[16] S. Srisakultiew and M. Siripruchyanun, "A Synthesis of Current-Mode PID Controller Using CCCIIs," The Proceeding of $3^{\text {rd }}$ National Conference on Technical Education, 2010, pp. 63-68.

[17] A. U. Keskin, "Design of a PID Controller Circuit Employing CDBAs," International Journal of Engineering Education, Vol. 43, No.1, 2006, pp. 48-56. doi:10.7227/IJEEE.43.1.5

[18] C. Acar and S. Ozoguz, "A New Versatile Building Block: Current Differ-Encing Buffered Amplifier Suitable for Analog Signal Processing Filters," Microelectronics Journal, Vol. 30, No. 2, 1999, pp. 157-160. doi:10.1016/S0026-2692(98)00102-5

[19] S. Ozoguz, A. Toker and C. Acar, "Current-Mode Continuous-Time Fully-Integrated Universal Filter Using CDBAs," Electronics Letters, Vol. 35, No. 2, 1999, pp. 97-98. doi:10.1049/el:19990118

[20] S. Maheshwari and I. A. Khan, "Current-Controlled Current Differencing Buffered Amplifier: Implementation and Applications," Active and Passive Electronic Components, Vol. 27, No. 4, 2004, pp. 219-227. doi:10.1080/08827510310001648924

[21] C. Toumazou, F. J. Lidgey and D. G. Haigh, "Analog IC Design: The Current Approach," Peter Peregrinus, London, 1990

[22] D. R. Frey, "Log-Domain Filtering: An Approach to Current-Mode Filtering," IEE Proceeding Circuit Devices System, Vol. 140, No. 6, 1993, pp. 406-416. 